GLOBAL WATER PATHOGEN PROJECT

PART FIVE. CASE STUDIES

\title{
USING GENETIC MICROBIAL SOURCE TRACKING (MST) MARKERS TO IDENTIFY FECAL POLLUTION SOURCES IN SPRING WATER OF A LARGE ALPINE KARST CATCHMENT
}

Rita Linke

Research Group of Environmental Microbiology and Molecular Diagnostics, Institute of Chemical, Environmental and Bioscience Engineering, TU Wien Vienna, Austria

P. Stadler

Centre for Water Resource Systems (CWRS), TU Wien

Vienna, Austria

\section{Georg H. Reischer}

Research Group of Environmental Microbiology and Molecular Diagnostics, Institute of Chemical, Environmental and Bioscience Engineering, TU Wien Molecular Diagnostics Group, Department IFA-Tulln

Vienna, Austria

\section{Savio}

Karl Landsteiner University of Health Science, Department for Water Quality and Health

Krems, Austria

\section{Kollanur}

Research Group of Environmental Microbiology and Molecular Diagnostics, Institute of Chemical, Environmental and Bioscience Engineering, TU Wien Vienna, Austria

\section{R. Mayer}

Research Group of Environmental Microbiology and Molecular Diagnostics, Institute of Chemical, Environmental and Bioscience Engineering, TU Wien InterUniversity Cooperation Centre for Water \& Health

Vienna, Austria

\section{R.L. Mach}

Institute of Chemical, Environmental and Bioscience Engineering, TU Wien

Vienna, Austria

\section{A.K.T. Kirschner}

Institute for Hygiene and Applied Immunology, Water Hygiene, Medical University of Vienna

Vienna, Austria

Regina Sommer

Institute for Hygiene and Applied Immunology, Water Hygiene, Medical University of Vienna

Vienna, Austria

Julia Derx

Institute of Hydraulic Engineering and Water Resources Management, TU Wien

Vienna, Austria

\section{H. Stadler}

Department for Water Resources Management, Institute for Water, Energy and Sustainability, Joanneum Research

Graz, Austria 


\section{(c) (1) (1)}

This publication is available in Open Access under the Attribution-ShareAlike 3.0 IGO (CC-BY-SA 3.0 IGO) license (http://creativecommons.org/licenses/by-sa/3.0/igo). By using the content of this publication, the users accept to be bound by the terms of use of the UNESCO Open Access Repository (http://www.unesco.org/openaccess/terms-use-ccbysa-en).

Disclaimer:

The designations employed and the presentation of material throughout this publication do not imply the expression of any opinion whatsoever on the part of UNESCO concerning the legal status of any country, territory, city or area or of its authorities, or concerning the delimitation of its frontiers or boundaries. The ideas and opinions expressed in this publication are those of the authors; they are not necessarily those of UNESCO and do not commit the Organization.

Citation:
Linke, R.B., Stadler, P., Reischer, G.H., Savio, D., Kollanur, D., Mayer, R., Mach, R.L., Kirschner, A.K.T., Sommer, R., Derx, J., Stadler, H. and Farnleitner, A.H. (2019). Using genetic microbial source tracking (MST) markers to identify fecal pollution sources in spring water of a large alpine karst catchment. In: J.B. Rose and B. Jiménez-Cisneros, (eds) Water and Sanitation for the 21st Century: Health and Microbiological Aspects of Excreta and Wastewater

Management (Global Water Pathogen Project). (S. Petterson and G. Medema (eds) Part 5: Case Studies), Michigan State University, E. Lansing MI, UNESCO. https://doi.org/10.14321/waterpathogens.82

Acknowledgements: K.R.L. Young, Project Design editor; Website Design: Agroknow (http://www.agroknow.com) 


\section{Summary}

\section{Highlights}

- Apply MST markers as E. coli does not provide information on fecal pollution sources

- Support water resource management (SDG 6.5) by genetic MST marker detection
- Identified animals instead of suspected human sewage disposal as emission sources Demonstrate

- how to generate robust MST information at head water catchments

- Tiered MST approach as interface between E. coli diagnostics and QMRA

\section{Graphical abstract}

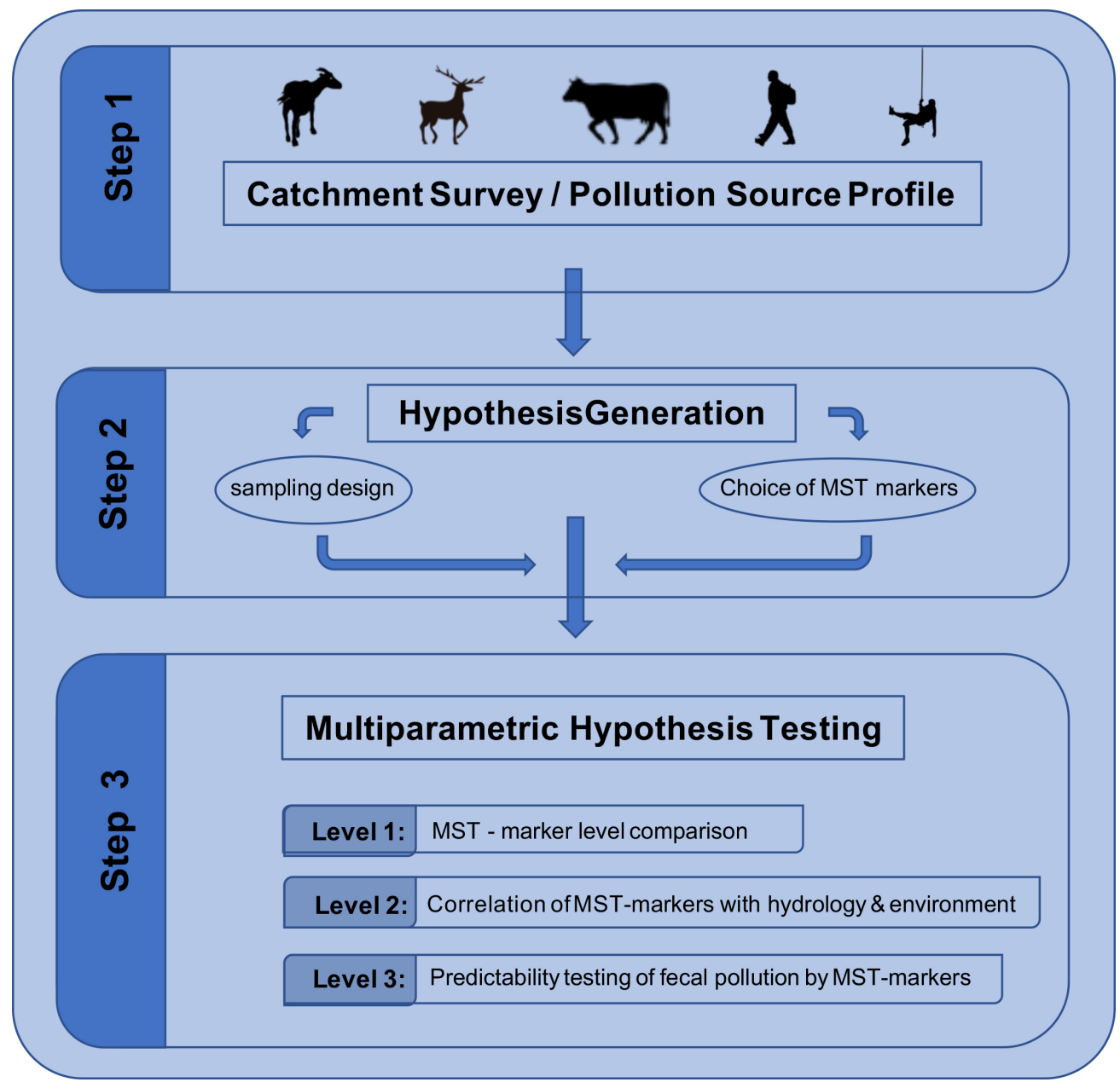

\section{Management objective}

Fecal pollution analysis still relies on cultivation-based E. coli and intestinal Enterococci (FIB) standard methods. However, these approaches do not give information on the kind of pollution sources. This study demonstrates how to identify pollution sources based on the application of genetic microbial source tracking (MST) markers at alpine spring water to guide target-oriented catchment protection and management.

\section{Location and description of the system}

The study site is located in the Northern Calcareous Alps (Austria) and drains a large Triassic limestone aquifer between 820 and $1828 \mathrm{~m}$ (catchment approx. $70 \mathrm{~km} 2$ ). Vegetation comprises summer pastures, natural calcareous alpine swards with open krummholz and forests. Potential fecal pollution sources include humans, livestock (cattle) and wild life. During base-flow conditions the observed spring has excellent water quality. In contrast, heavy rain events can result in high FIB concentrations. 


\section{Description of the method}

The integrated multi-tiered approach covers, i) pollution source profiling using information from catchment survey activities, ii) hypothesis formulation on the potentially important fecal pollution sources and MST assay selection, and finally iii) hypothesis testing at the spring using hydrology-driven sampling and data stratification with concurrent analysis of FIB and genetic MST markers.

\section{Outcome and recommendations}

- FIB could be traced back to animal fecal pollution sources (ruminants). Despite of being initially suspected, human sewage was not identified as the source of contamination.

- Results verified catchment protection measures and also guided further hazard and risk assessment activities.

- The tiered approach proved very valuable for MST. It is also suitable for the application in other complex headwater catchments, since the used parameters and methods can be adapted to the specific situation.

\section{Introduction}

Alpine and mountainous karstic springs are of high importance for drinking water supply worldwide (Ford and Williams, 2007). It is estimated that nearly a quarter of the world's population is dependent on groundwater from karstic aquifers, including alpine, mountainous and low land karstic systems. In most cases, alpine karstic systems offer good raw-water quality under base flow conditions (Farnleitner et al., 2005; Wilhartitz et al., 2009). However, they are frequently very susceptible to precipitation events and a rapid decrease in water quality can often be observed during such situations. Microbial fecal pollution and input of fecal associated pathogens via rapid surface runoff, through temporarily activated karst conduits, is the most abundant quality hazard (Ford and Williams 2007; Savio et al.,2018). Thus, water resources from (alpine) karst aquifers require sustainable protection from fecal pollution within the catchment and proper microbial quality management at and after the spring (e.g. optimized spring water abstraction, adjusted treatment and disinfection measures) if used for drinking water supply (Farnleitner et al., 2018; Savio et al., 2018).

In practice, microbiological spring water quality is monitored by the application of standardized methods to enumerate fecal indictor bacteria (SFIB), most frequently using E. coli and/or intestinal enterococci as target organisms. Cultivation-based enumeration of SFIB is still regarded the "gold standard" for many water resources in order to sensitively evaluate the extent of fecal contamination (Farnleitner et al., 2010). However, detection of SFIB in water resources does not give any information on the origin of the contamination source, because SFIB do occur in wildlife, livestock and human sources (Farnleitner et al., 2010; Frick et al., 2018).

The aim of this case study is to demonstrate how SFIB spring monitoring can be complemented by applying hostassociated microbial source tracking (MST) markers to identify responsible fecal sources in case of contamination. Genetic MST markers are determined by state-of-the-art quantitative PCR (qPCR) methodology. A robust and straightforward methodology ("hypothesis-driven approach") is presented for the investigated dynamic alpine karstic water resource, integrating best available information from the catchment with monitoring efforts from the spring environment.

\section{Problem formulation}

During base-flow conditions the investigated spring (limestone karst aquifer spring number 6; LKAS6) shows good raw water quality (Farnleitner et al., 2005; Wilhartitz et al., 2009) and - based on SFIB enumeration - microbial water quality problems do apparently not exist (SFIB are not detectable in the applied monitoring volume). Detection of fecal pollution is limited to the warm season and increased discharge situations (e.g. snow-melt, heavy rain events during summertime). For such periods, moderate to strong fecal pollution events are reported (Stadler et al., 2008; Farnleitner et al., 2011). For example, the herein presented investigation period showed pollution events in the range of $\log 10$ 1.1-4.0 and of $\log 10$ 0.3-3.8 CFU per $100 \mathrm{ml}$ for E. coli and enterococci, respectively. However, no information on the responsible fecal contamination sources was available before the application of the herein presented approach. Nevertheless, this information is considered of highest relevance for hazard- and health risk assessment and to guide target-oriented catchment management and best environmental practices.

\section{Description of the system, nested sampling and basic parameters}

The selected study site is located in the Northern Calcareous Alps (Austria) and drains a large Triassic limestone aquifer between 820 and $1828 \mathrm{~m}$ (catchment approx. $4 \mathrm{~km} 2$ ). The spring, LKAS6, shows a pronounced variation in flow conditions, with a mean discharge of 244

L.s-1 (range: 118 to 1150 L.s-1). Vegetation in the investigated area comprises summer pastures, natural calcareous alpine swards with open krummholz and forests (Grabherr et al., 1999). Several mountain lodges, supporting touristic activities, also exist (Reischer et al., 2011). 
Hydrological characterization of the spring was achieved by collecting data on discharge, temperature, electrical conductivity, spectral absorption coefficient at $254 \mathrm{~nm}$ (SAC254), and turbidity measured by infield online sensors directly installed at the outlet of the spring (Farnleitner et al., 2005; Stadler et al., 2008,;Stadler et al., 2010).

Considering hydrological discharge dynamics, a nested sampling approach was followed resulting in a total of three sampling tiers. A basic monitoring sampling (BMS) was performed on a 3- to 4-weeks interval from June 2007 to November 2008 and a high-frequency sampling (HFS) ). during summer time (i.e., period of increased hydrologicaland fecal pollution dynamics) was performed twice a week. Furthermore, one flood event (EVENT08) was analyzed in detail during late summer 2008 (cf. Figure 1

SFIB (E. coli, enterococci), presumptive Clostridium perfringens, aerobic spore-forming bacteria and heterotrophic plate count bacteria at $22^{\circ} \mathrm{C}$ (HPC22) were performed for BMS, HFS and EVENT08 according to ISO standard methods (ISO 2000a; ISO 2000b; ISO 2002).

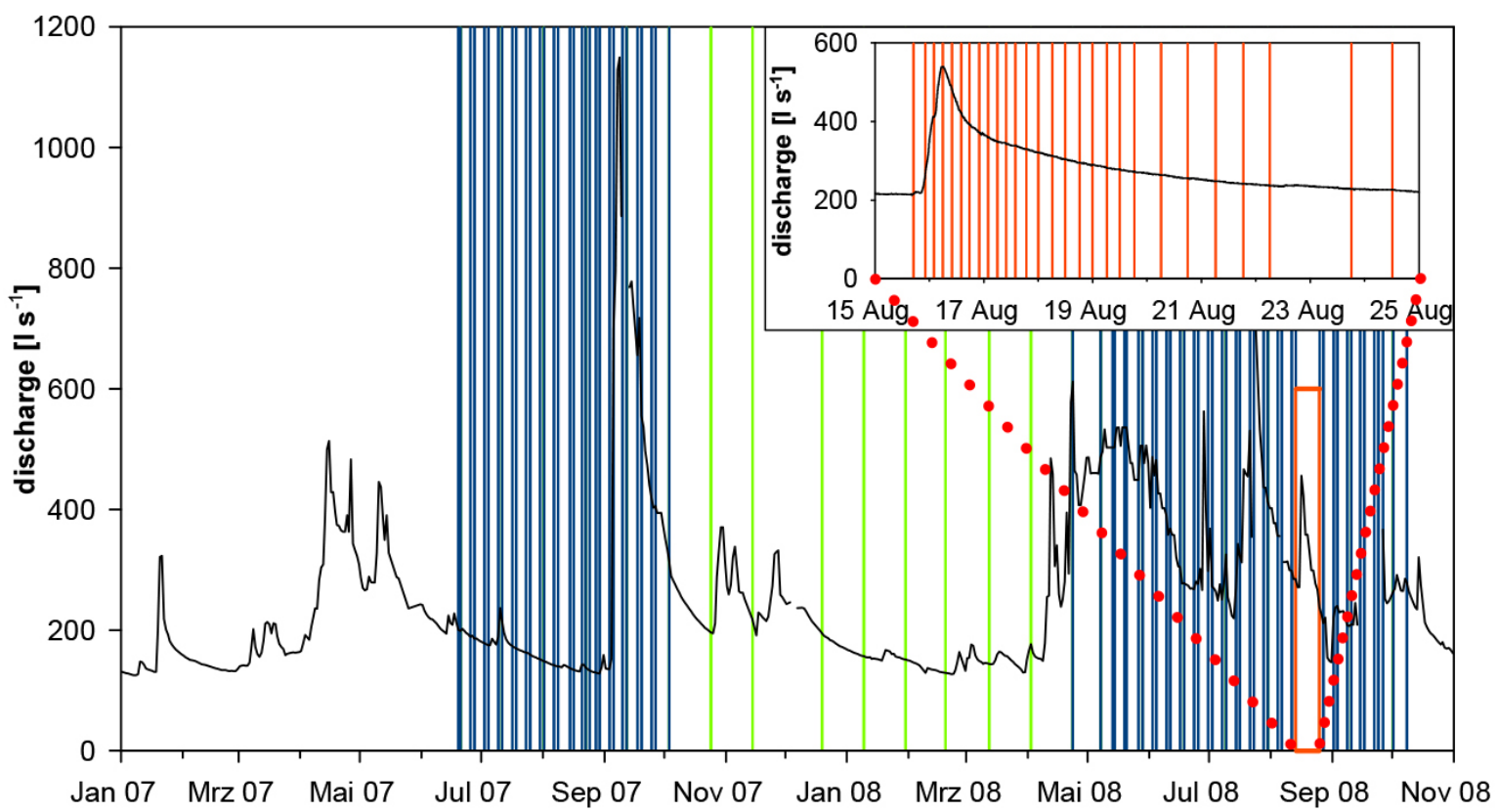

Figure 1: Hydrological situation of the spring and the applied "nested" sampling scheme. Discharge levels in the main graph show the discharge throughout the years 2007 and 2008 as daily mean values. Vertical lines mark the sampling dates during the monitoring program, green lines mark basic monitoring sampling dates, blue lines mark high frequency monitoring sampling dates during summer months, red box represents the flood event time frame, which is zoomed-in (upper right corner, linked with red dotted lines to the red flood event box), red lines within the high-resolution time scale mark event based high frequency monitoring sampling dates; discharge levels in the zoomed-in box are quarter-hourly measured values; gaps in the discharge data were due to malfunctions of the instruments necessary for the calculation of the discharge. Source: Farnleitner et al., 2011. 


\section{Description of the presented approach to complement SFIB monitoring with MST}

The presented concept is based on a three-step approach (Farnleitner et al., 2011; Reischer et al., 2013). STEP 1) A catchment survey to identify and characterize potential pollution sources - referred to as pollution source profiling (PSP; compare graphical abstract) - is undertaken. Visual inspection tours are organized to identify fecal pollution sources in the catchment. Further information for quantitative characterization of identified fecal sources is obtained from local authorities, official records, and scientific literature. Furthermore, information on the occurrence of SFIB from a broad range of animal and human sources in the catchment area was available and thus the amount of produced E. coli per source group could be estimated by the amount of environmentally available fecal material per source group $\mathrm{x}$ source group specific average E. coli concentrations in excreta or sewage (Farnleitner et al., 2011). STEP 2) Based on SFIB pollution load estimates, a hypothesis can be generated on the most likely pollution source(s) for the considered spring water habitat. The generated hypothesis then leads to the required sampling design and the choice of MST-markers to be applied. In the present study, a nested sampling design resulting in a total of three sampling tiers was chosen considering the hydrological discharge dynamics of the system (compare section: description of the system and applied methods; Fig. 1). STEP 3) The approach is completed by rigorous testing of the formulated hypothesis at the spring water environment by the retrieved information. For this, the generated MST-data is analyzed and discussed at 3 levels. (3.1) Comparison of the host-associated MST-maker levels in the spring water (i.e. human vs. animal marker) in context of the applied sampling regimes (here: BMS, HFS, EVENT08). (3.2) Statistical evaluation on existing associations between the MST-marker with the prevailing spring water quality situation by integrating all available hydrological, chemical, physical and microbiological parameters (i.e. multiparametric correlation analysis). (3.3) Finally (if applicable), testing the statistical predictability of SFIB pollution by the determined MSTmarker concentrations.

\section{Outcome}

STEP 1 (pollution source profiling at the catchment). Before the systematic investigation, humans were suspected the major source of fecal pollution due to the presence of significant touristic activities in the area (waste water, cesspits and/or illegal turnout thereof). Cattle grazing on pastures were assumed to play a minor role.
Results from the PSP, however, identified ruminants (cattle and wildlife) as the most important potential source of fecal pollution. Their daily production of environmentally available fecal matter was estimated to amount to 3.6 tons (equivalent to an E. coli load of $5.2 \times 1013 \mathrm{CFU} / \mathrm{d}$ ) in the catchment area (Farnleitner et al., 2011). Environmentally available fecal matter from human sources, in contrast, was negligible despite the presence of significant touristic activities in the area $(0.04$ $\mathrm{kg}$ wet weight/day; equivalent to an E. coli load of $3.6 \times 109$ CFU/d; Farnleitner et al., 2011). This was attributed to the fact that sufficient sanitary facilities at restaurants and lodges exist which reduce direct fecal pollution from hikers and climbers to a minimum (i.e. low environmental availability of the produced fecal material). Moreover, state-of-the-art sewage disposal facilities at restaurants and lodges further minimize the input of fecal pollution of human origin to the environment.

STEP 2 (hypothesis generation and study design formulation). From PSP results, it was hypothesized that ruminants instead of originally suspected human fecal excreta and sewage were responsible for the occasionally observed high levels of fecal indicator bacteria in the spring water of LKAS6. Based on these findings MST-tools to detect human and ruminant associated fecal pollution were chosen to test this hypothesis. To target fecal pollution of human origin the BacH assay (Reischer et al., 2007) was chosen and for the detection of fecal pollution originating from ruminants (wildlife and cattle) the BacR assay (Reischer et al., 2006) was chosen. Both assays target Bacteroidetes populations associated with their hosts. Concerning performance criteria of MST-tools, it has been suggested that no fixed performance thresholds categorize an assay as suitable/applicable (Harwood et al., 2011; Belanche et al., 2011). Instead, to ensure applicability of a MST assay its performance must match the requirements for each MST problem and the conditions within the study area specifically (Reischer et al., 2013). For the investigated LKAS6 catchment, Reischer et al., (2011) used an approach based on Bayes' theorem to estimate the probability for the correct detection of a specific source of pollution under different pollution scenarios (Reischer et al. 2011; Kildare et al., 2007). In addition, both, the BacH and BacR assay, were tested for their level of specificity and sensitivity for the respective fecal source group(s) on samples from the LKAS6 catchment.

STEP 3 (multi-parametric hypothesis testing at the spring environment) All microbiological and most of the physicochemical parameter values showed strong variations throughout the year and the nested sampling design nicely covered the system dynamics (Fig. 1). 
Level 1 - analysis: Regarding the results of the genetic Bacteroidetes markers associated with either human $(\mathrm{BacH})$ or ruminant (BacR) fecal pollution, BacR marker levels were found to be several orders of magnitude higher than those of $\mathrm{BacH}$, irrespective of the sampling tier investigated (Fig. 2). BacH levels were frequently close to or below the detection threshold. Since $\mathrm{BacH}$ marker concentration in human fecal material was shown to be around 1 order of magnitude higher than BacR marker concentrations in ruminant feces the $\mathrm{BacH}$ marker concentrations have additionally been corrected for the higher abundance to allow a quantitative direct comparison of fecal input from human versus ruminants in spring water (BacH corr., Fig. 2). The finding of higher levels of BacR clearly supports the hypothesis that ruminants (cattle and wildlife) instead of humans were the most abundant source of fecal pollution in the catchment area.

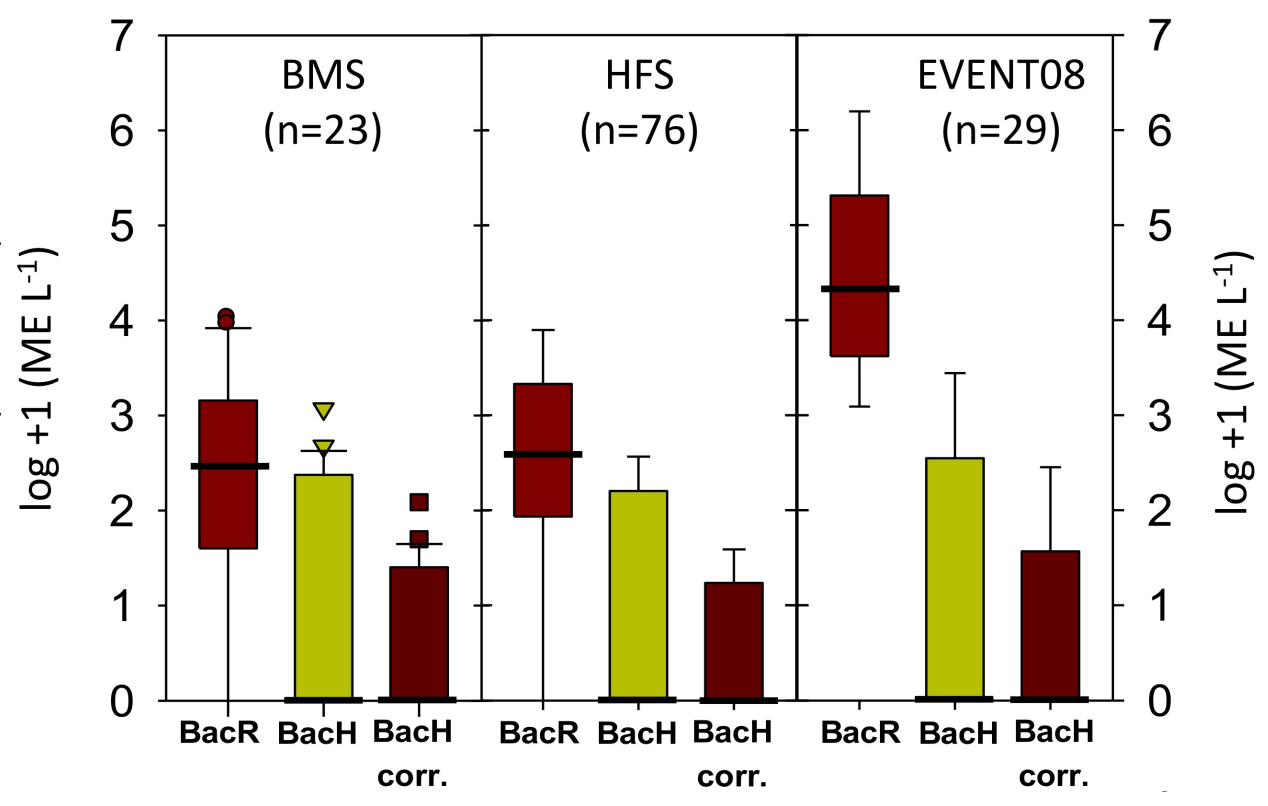

Figure 2. Abundances of the BacR and BacH marker in LKAS6 during basic monitoring sampling (BMS), high frequency sampling (HFS) and flood event monitoring (EVENT08). Box plots show the distribution of BacR and BacH marker values (whiskers, 10th and 90th percentile; boxes, 25th and 75th percentile; bold lines within the box, median). BacR, ruminantassociated marker (red boxes and dot symbols); BacH, human-associated marker (green boxes and triangle symbols); BacH corr., human-associated marker after correction for the higher abundance in feces as compared to ruminant marker. Data is directly derived from BacH results after consideration of the correction factor (0.10); ME, marker equivalent; $\mathbf{n}$, number of samples; data is given after log+1- transformation. Source: modified from Farnleitner et al., 2011.

Level 2 - analysis: Among all investigated parameters, BacR revealed the highest association with E. coli and enterococci (ENT). It is interesting to note that correlation coefficients between BacR and SFIB increased with the rising hydrological dynamics. For example, correlation coefficients between BacR and ENT increased from 0.59 to 0.80 and 0.96 for the BMS, the HFS and the EVENT08 sampling, respectively (Tab. 1). This effect can be explained by the different persistence of applied markers and indicators becoming less influential with decreasing average residence times of the water masses (i.e., less time available for different die-off or degradation effects). In contrast to BacR, BacH did not show any relevant correlations with E. coli or ENT. 


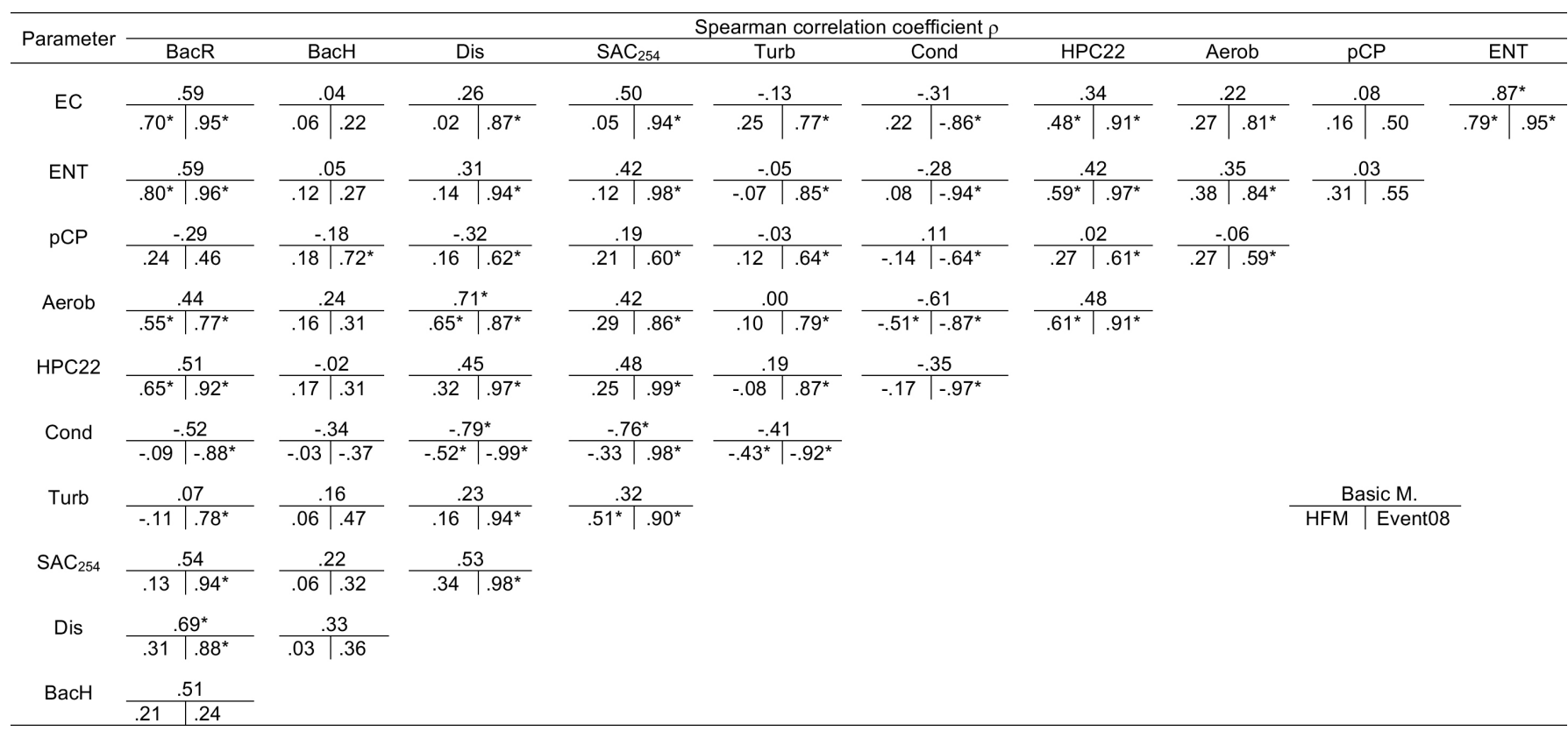

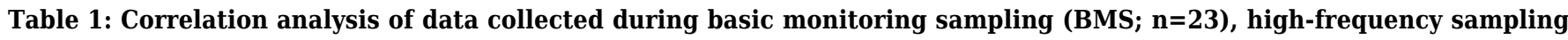
(HFS; $\mathbf{n = 7 5 - 7 6 )}$ and flood event 2008 based sampling (EVENT08; $n=29$ ). Abbreviations: Aerob: aerobic spore formers, BacH: human-associated genetic Bacteroidetes marker, BacR: ruminant-associated genetic Bacteroidetes marker, Cond: conductivity, Dis: discharge, EC: E. coli, ENT: enterococci, HPC22: heterotrophic plate count at $22^{\circ} \mathrm{C}$, pCP: presumptive Clostridium perfringens, SAC254: spectral absorption coefficient at $254 \mathbf{~ n m}$, Turb: turbidity. Source: Farnleitner et al., 2011.

Level 3 - analysis: E. coli and BacR were selected for further statistical analysis due to the high identified association between these parameters (see level 2). Using regression analysis for the parameters E. coli and BacR, it was able to predict $90 \%$ of the E. coli variation using BacR values (Fig. 3), providing strong support that E. coli was predominantly derived from ruminant fecal pollution sources (Farnleitner et al., 2011). $\mathrm{BacH}$ could not be used for regression analysis as indicated by the correlation analysis above, with most values being below the level of detection. 


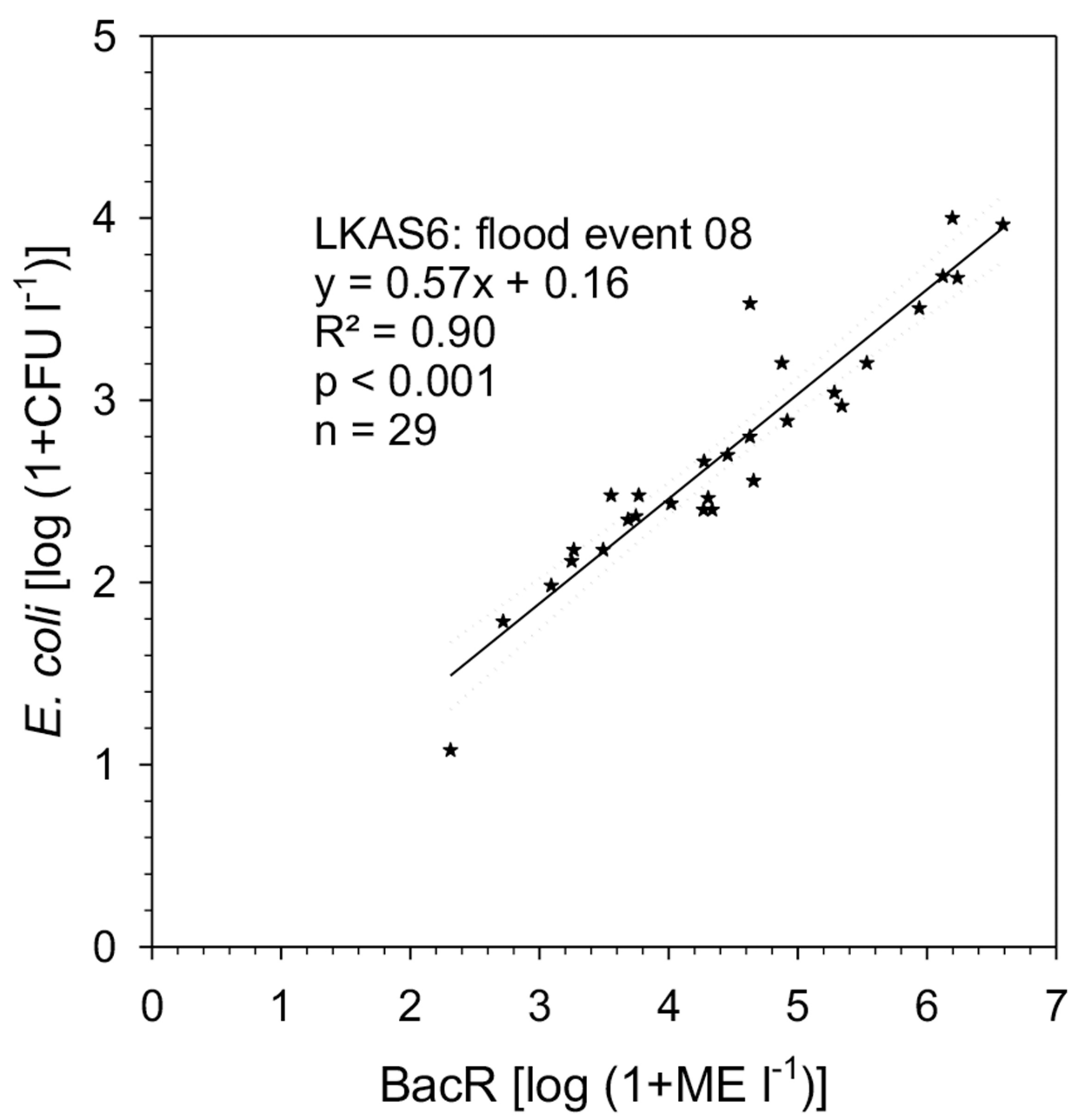

Figure 3. Regression analysis of BacR and E. coli data of flood event 2008 in LKAS6. BacR, ruminant-specific marker; ME, marker equivalents; CFU, colony forming units; solid line, regression curve; $\mathbf{R}^{2}$, coefficient of determination for linear regression; data is given after $\log 10$-transformation and addition of 1 to a given value. Source: Farnleitner et al., 2011

In conclusion, all 3 levels of analysis indicated that ruminant instead of previously suspected human sources were responsible for the occasionally observed high levels of SFIB in spring water of the LKAS6 catchment.

\section{Implications for practitioners and catchment and risk management}

In this case study, we introduced a robust and relatively simple approach to guide environmental authorities and water utilities for pollution source identification in case standard fecal indicators (E. coli and enterococci) are detected in spring water. Microbial source tracking by using host-associated bacterial genetic fecal makers was successfully applied as a supporting technique to complement standardized fecal indicator bacteria assays to answer the crucial question which source(s) of fecal pollution had to be considered (human vs. livestock, wildlife) in the catchment. By this approach, the "black box" of fecal pollution can be opened and responsible sources can be unraveled in the catchment area (Savio et al., 2018).
The presented concept based on Pollution Source Profiling (PSP), hypothesis formulation, definition of MST requirements and hypothesis testing, is also easily applicable for other types of "headwater" catchments and not restricted to alpine karst water resources. Complementation with standard methods is easy to perform by filtering additional water samples in addition to SFIB determination. The filters can be stored $\left(-80^{\circ} \mathrm{C}\right)$ and analyzed in case needed (e.g. periodic detection of fecal pollution). Numbers and types of applicable MST assays available in the literature are increasing rapidly, reducing limitations due to lacking assays for specific sources and/or habitats. Identifying the sources of fecal pollution in the water resource, as originally determined by standardized SFIB assays, can be an essential part for system assessment (i.e. water safety planning, sanitation safety planning), target-oriented catchment management, and finally, can serve also as a very important starting point for hazard- and risk assessment and MST-guided QMRA (Savio et al., 2018). 


\section{Acknowledgements}

This case study was derived from a previous research project, the results of which are published in the following book chapter:
This paper was supported by Vienna Water (MA31) and the Science Call 2015 "Ressource und Lebensgrundlage Wasser" Projekt SC15-016, supported by the Niederösterreichischen Forschungs- und Bildungsgesellschaft (NFB). This is a joint study effort of the ICC Water \& Health (www.waterandhealth.at).

Farnleitner, A. H., Reischer, G. H., Stadler, H., Kollanur, D., Sommer, R., Zerobin, W., Blöschl, G., Barrella, K. M., Truesdayle, J. A., Casarez, E. A. \& Di Giovanni, G. D. (2011). Agricultural and rural watersheds. In Microbial Source Tracking: Methods, Applications, and Case Studies (pp. 399-431). Springer, New York, NY. https://link.springer.com/book/10.1007/978-1-4419-9386-1 


\section{References}

Belanche, L.A., Blanch, A.R., Hagedorn, C., Blanch, A.R. and Harwood, V.J. (2011). Statistical Approaches for Modeling in Microbial Source Tracking. Microbial Source Tracking: Methods, Applications, and Case Studies. Springer. New York. pp. 207-277.

Farnleitner, A.H., Reischer, G.H., Stadler, H., Kollanur, D., Sommer, R., Zerobin, W. et al. (2011). Agricultural and rural watersheds. Microbial Source Tracking: Methods, Applications, and Case Studies. Springer. New York. pp. 399-431.

Farnleitner, A.H., Ryzinska-Paier, G., Reischer, G.H., Burtscher, M.M., Knetsch, S., Kirschner, A.K. et al. (2010). Escherichia coli and enterococci are sensitive and reliable indicators for human, livestock and wildlife faecal pollution in alpine mountainous water resources. Journal of applied microbiology. 109, pp. 1599-1608.

Farnleitner, A.H., Savio, D., Sommer, R., Reischer, G., Kirschner, A., Zerobin, W. et al. (2018). Integrated strategy to guide health-related microbial quality management at alpine karstic drinking water resources. In Karst Groundwater Contamination and Public Health. Springer International Publishing. pp. 185-192.

Farnleitner, A.H. and Wilhartitz, I.C. (2005). Bacterial dynamics in spring water of two contrasting alpine karst aquifers indicate autochthounous microbial endokarst communities. Environmental Microbiology. 7, pp. 1248-1259.

Ford, D.C. and Williams, P. (2007). Karst hydrogeology and geomorphology. Wiley. New York.

Frick, C., Vierheilig, J., Linke, R., Savio, D., Zornig, H., Antensteiner, R. et al. (2018). Poikilothermic animals as a previously unrecognized source of fecal indicator bacteria in a backwater ecosystem of a large river. Applied and environmental microbiology. AEM-00715, doi: 10.1128/AEM.00715-18.

Grabherr, G.T., Dirnbock, T., Dullinger, S. and Gottfried, M. (1999). Vegetationskartierung Hochschwab-Aflenzer Staritzen. Institute of Plant Physiology, Vienna University. Vienna.

Harwood, V.J. and Stoeckel, D.M. (2011). Performance Criteria. Microbial Source Tracking: Methods, Applications, and Case Studies ed. (Hagedorn, C. and Blanch, A.R., ed.). Springer. New York.

ISO (2002). Water Quality - Detection and Enumeration of Clostridium perfringens - Part 2: Method by Membrane filtration (ISO/CD 6461-2). International Organization of Standarization. Geneva, Switzerland.

ISO (2000). Water Quality - Detection and Enumeration of Intestinal Enterococci - Part 2: Membrane Filtration Method (ISO 7899-2: 2000). International Organization of Standardization. Geneva, Switzerland.

ISO (2000). Water Quality - Detection and Enumeration of Escherichia coli and Coliform Bacteria - Part 1: Membrane Filtration Method (ISO 9308-1: 2000). International Organization of Standardization. Geneva, Switzerland.

Kildare, B.J., Leutenegger, C.M., McSwain, B.S., Bambic, D.G., Rajal, V.B. and Wuertz, S. (2007). 16S rRNA-based assays for quantitative detection of universal, human-, cow-, and dog-specific fecal Bacteroidales: a Bayesian approach. Water research. 41(16), pp. 3701-3715.

Reischer, G.H., Ebdon, J.E., Bauer, J.M., Schuster, N., Ahmed, W., Astrom, J. et al. (2013). Performance characteristics of qPCR assays targeting human-and ruminant-associated Bacteroidetes for microbial source tracking across sixteen countries on six continents. Environmental science and technology. 47, pp. 8548-8556.

Reischer, G.H. and Kasper, D.C.et al (2007). A quantitative real-time PCR assay for the highly sensitive and specific detection of human faecal influence in spring water from a large alpine catchment. Letters in Applied Microbiology. 44, pp. $351-356$.

Reischer, G.H. and Kasper, D.C.et al. (2006). Quantitative PCR method for sensitive detection of ruminant faecal pollution in freshwater and evaluation of this method in alpine karstic regions. Applied Environmental Microbiology. 72, pp. 5610-5614. 

Reischer, G.H. and D. Kollanur, et.al. (2011). A hypothesis-driven approach for the identification of fecal pollution sources in water resources. Environmental Science and Technology. 45, pp. 4038-4045.

Savio, D., Stadler, P., Reischer, G.H., Kirschner, A.K., Demeter, K., Linke, R. et al. (2018). Opening the black box of spring water microbiology from alpine karst aquifers to support proactive drinking water resource management. Wiley Interdisciplinary Reviews: Water. 5, pp. e1282.

Stadler, H., Klock, E., Skritek, P., Mach, R.L., Zerobin, W. and Farnleitner, A.H. (2010). The spectral absorbance coefficient at $254 \mathrm{~nm}$ as a near real time early warning proxy for detecting faecal pollution events at alpine karst water resources. Water Science and Technology. 62, pp. 1898-1906.

Stadler, H. and P. Skritek, et.al. (2008). Microbiological monitoring and automated event sampling at karst springs using LEO-satellites. Water Science Technology. 58, pp. 899-909.

Wilhartitz, I.C., Kirschner, A.K., Stadler, H., Herndl, G.J., Dietzel, M., Latal, C. et al. (2009). Heterotrophic prokaryotic production in ultraoligotrophic alpine karst aquifers and ecological implications. FEMS microbiology ecology. 68(3), pp. 287-299. 